\title{
Evidence From HETE-2 For GRB Evolution With Redshift
}

\author{
Carlo Graziani*, Donald Q. Lamb*, Takanori Sakamoto ${ }^{\dagger * *}$, Timothy \\ Donaghy*, Jean-Luc Atteia ${ }^{\ddagger}$ and The HETE-2 Science Team ${ }^{\S}$ \\ ${ }^{*}$ Department of Astronomy \& Astrophysics, University of Chicago \\ ${ }^{\dagger}$ Department of Physics, Tokyo Institute of Technology \\ ${ }^{*}$ RIKEN (Institute of Physical and Chemical Research) \\ ${ }^{*}$ Centre D’Etude Spatiale des Rayonnements, France

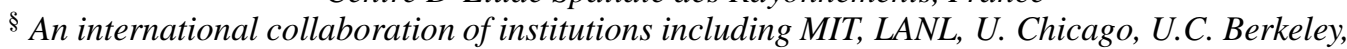 \\ U.C. Santa Cruz (USA), CESR, CNES, Sup'Aero (France), RIKEN, NASDA (Japan), IASF/CNR \\ (Italy), INPE (Brazil), TIFR (India)
}

\begin{abstract}
.
After taking into account threshold effects, we find that the isotropic-equivalent energies $E_{\text {iso }}$ and luminosities $L_{\text {iso }}$ of gamma-ray bursts (GRBs) are correlated with redshift at the $5 \%$ and $0.9 \%$ signficance levels, respectively. Our results are based on 10 BeppoSAX GRBs and 11 HETE-2 GRBs with known redshifts. Our results suggest that the isotropic-equivalent energies and luminosities of GRBs increase with redshift. They strengthen earlier clues to this effect from analyses of the BATSE catalog of GRBs, using the variability of burst time histories as an estimator of burst luminosities (and therefore redshifts), and from an analysis of BeppoSAX bursts only. If the isotropic-equivalent energies and luminosities of GRBs really do increase with redshift, it suggests that GRB jets at high redshifts may be narrower and thus the cores of GRB progenitor stars at high redshifts may be rotating more rapidly. It also suggests that GRBs at very high redshifts may be more luminous - and therefore easier to detect - than has been thought, which would make GRBs a more powerful probe of cosmology and the early universe than has been thought.
\end{abstract}

\section{INTRODUCTION}

GRB sources are a cosmologically-distributed population. Like all other such populations, their properties presumably evolve with redshift. In this paper, we address two key questions: what evidence exists bearing on the evolution of GRB energetics as a function of redshift, and what is the nature and magnitude of that evolution?

\section{PREVIOUS INDICATIONS OF GRB EVOLUTION}

Using a Cepheid-like variability-based redshift measure developed together with Fenimore \& Ramirez-Ruiz [4], Lamb \& Reichart [5] found evidence for a positive correlation between isotropic-equivalent peak luminosity $L_{\text {iso }}$ and redshift, using BATSE data. The left panel of Figure 1 shows their variability-based luminosity estimator, plotted as a function of redshift. The diagonal lines represent the BATSE 10\% and 90\% detection thresholds. 

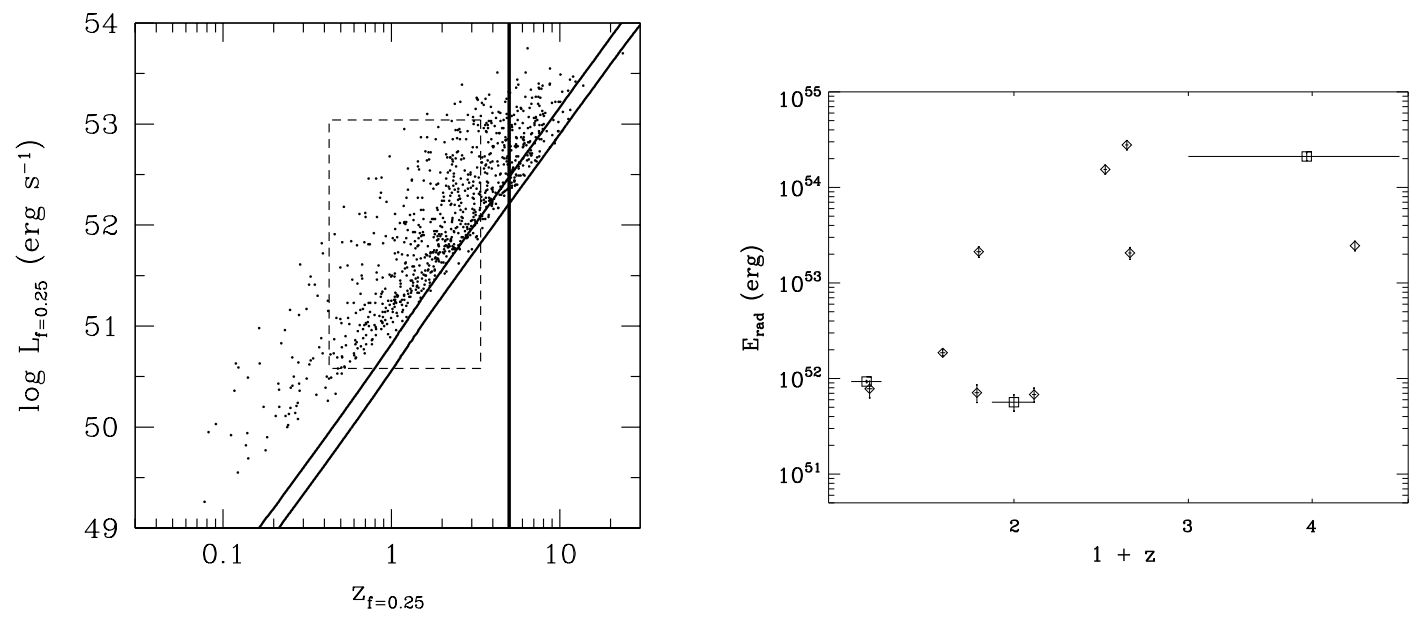

FIGURE 1. Left Panel: Variability Measure of Luminosity versus redshift. Lamb \& Reichart [5]. Right panel: $E_{\text {iso }}$ versus redshift for BeppoSAX GRB afterglows with redshift determinations. Amati et al. [1].

As can be seen in the figure, there is a dearth of high-luminosity bursts at estimated redshifts below $z \sim 1$ and the estimated luminosities also "peel off" the threshold at estimated redshifts below $z \sim 1$, hinting at a correlation of burst luminosity with redshift. Nevertheless, threshold effects, together with the possibility of unknown systematic effects intrinsic to the variability measure, are causes for concern.

Lloyd-Ronning, Fryer, \& Ramirez-Ruiz [6] addressed threshold truncation effects in a study of 220 BATSE GRBs. They found that after applying the correction, there was significant evidence for a correlation slope $L_{\text {iso }} \sim(1+z)^{1.4}$.

The number of GRBs with known redshifts has now grown to the point that it is possible to do meaningful studies of the distributions of GRBs with spectroscopic redshifts, as opposed to the less certain redshifts derived from the GRB variability measure. Amati et al. [1], using a sample of 12 BeppoSAX GRBs with afterglows and redshift estimates, found a positive correlation between the isotropic-equivalent prompt energy $E_{\mathrm{iso}}$ and redshift, with a reported significance of $7 \%$. The right panel of Figure 1 shows the data used by Amati et al. [1]. While the result is promising, threshold effects were not quantified, and the distribution of $L_{\text {iso }}$ was not investigated.

The HETE-2 bursts increase the size of the sample of GRBs with known redshifts by a factor of 2, and therefore offer the opportunity to confirm (or possibly refute) the Amati et al. results. In addition, it is interesting to extend the Amati et al. results to isotropicequivalent peak luminosities $\left(L_{\text {iso }}\right)$, to make contact with the results of Lamb \& Reichart [5] and of Lloyd-Ronning, Fryer, \& Ramirez-Ruiz [6].

\section{THE HETE-2-AUGMENTED GRB SAMPLE}

The HETE-2 events considered in this study are:

- 9 HETE-2-localized classical GRBs with afterglows and redshifts — GRB010921, 

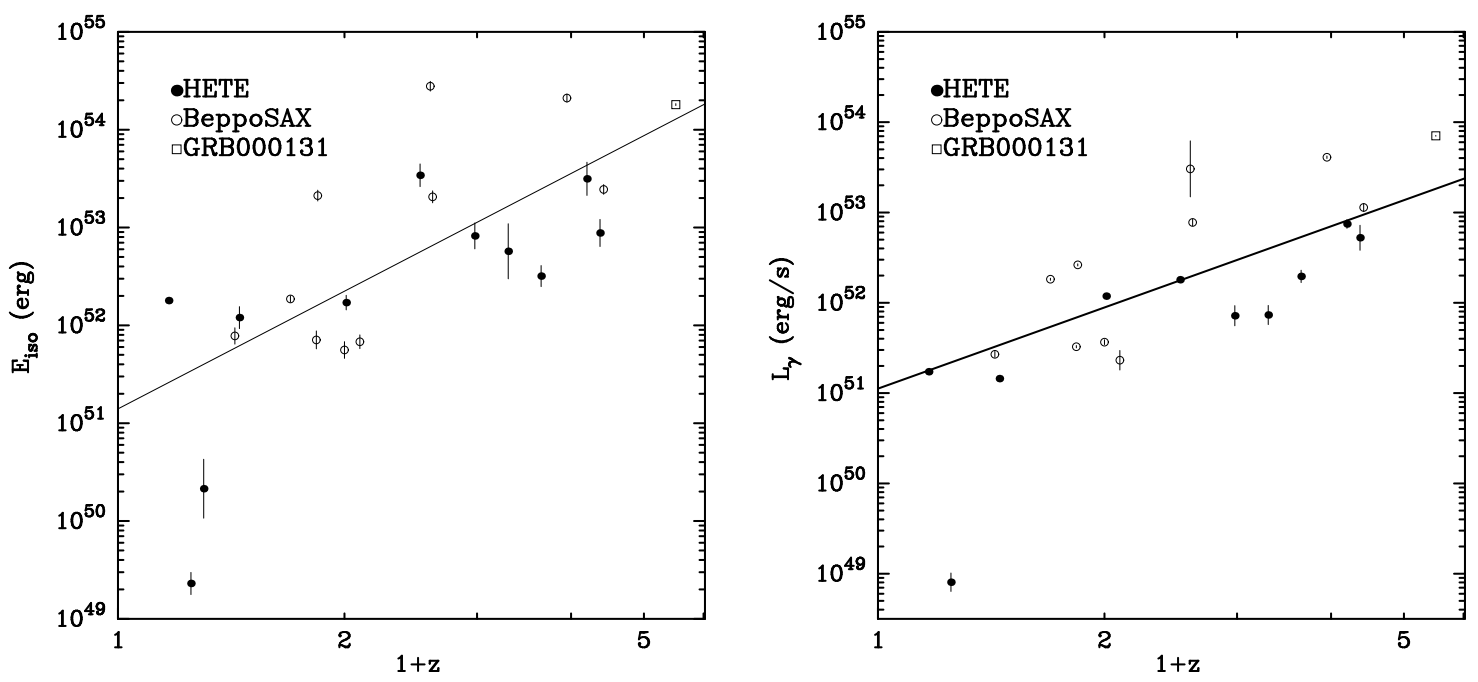

FIGURE 2. Left Panel: Isotropic-equivalent energy versus redshift. Right Panel: Isotropic-equivalent Luminosity versus redshift.

GRB020124, GRB021004, GRB021211, GRB030226, GRB030328, GRB030329, GRB030429, and GRB030323.

- 2 HETE-2-localized XRFs with afterglows and redshifts - XRF020903 $(z=0.25)$, and XRF030723 $(z \lesssim 0.3)$.

In addition to these eleven events, we consider GRB000131 $(z=4.511)$, an IPNlocated, BATSE-detected GRB. We further consider 10 events with well-determined redshifts from the Amati et al. [1] sample. In treating these events, we extract values of $L_{\text {iso }}$ (not otherwise reported in [1]) using the values of $E_{\text {iso }}, S$, and $F_{\text {peak }}$ provided in their paper, with due attention to cosmological corrections.

The data are shown in Figure 2 The straight lines show fits obtained by excluding the two HETE-2-located XRFs, which would otherwise obviously bias the fits toward very high slopes, whose value for extrapolation to high- $z$ would be dubious.

\section{ANALYSIS}

We perform a linear-regression analysis to the isotropic-equivalent prompt energy and peak luminosity data, obtaining best-fit slopes and intercepts. We also calculate correlation coefficients, and evaluate the formal correlation significance using the standard $t$-test.

This formal significance does not account in any way for threshold effects. We address the question of threshold-related systematic distortions by calculating detection thresholds using the methods described in Band [2]. The thresholds corresponding to WXM and to BeppoSAX are shown by the dashed lines in Figure 3.

We calculate threshold-corrected correlation significance by generating simulated samples of BeppoSAX- and HETE-2-observed data, which are truncated by the thresholds. The key assumption we make in the simulations is that the burst luminosity func- 

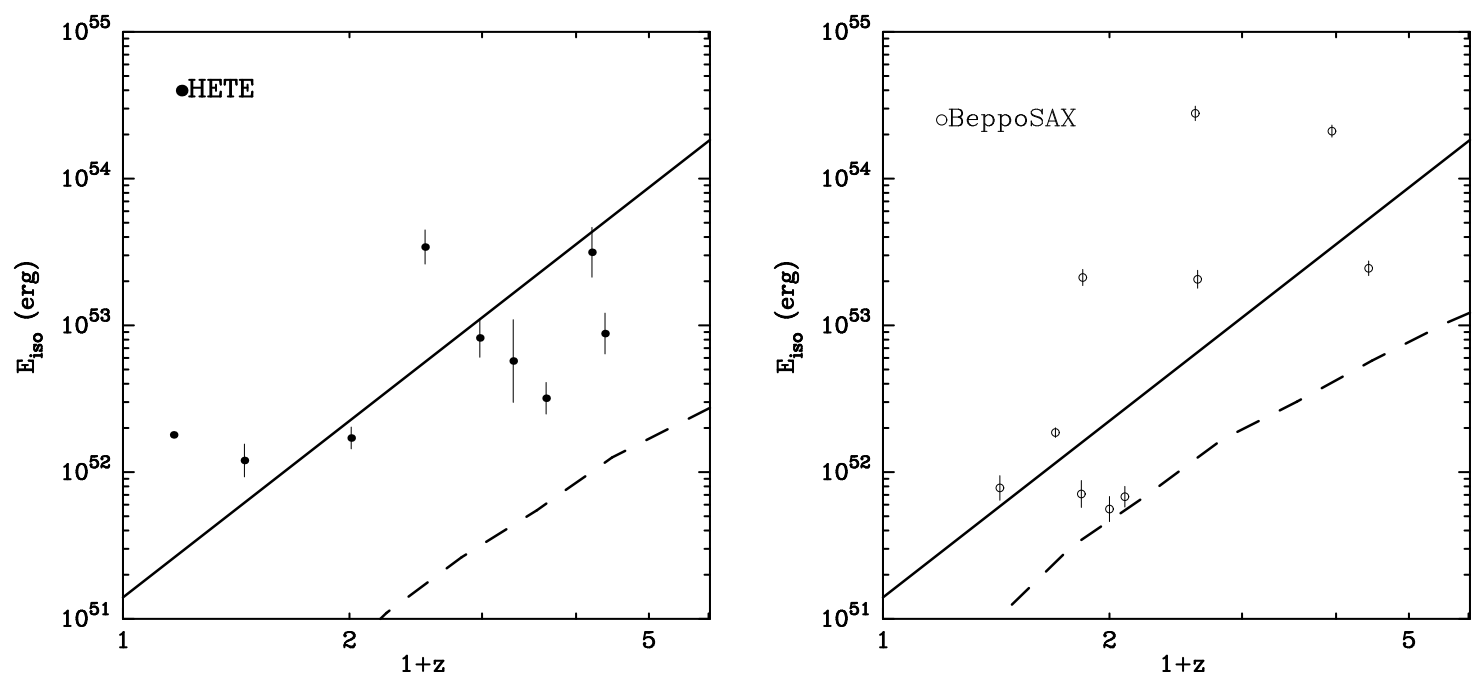

FIGURE 3. Isotropic-equivalent energy versus redshift. The dashed line shows the estimated detection threshold, calculated using the results in [2]. Left Panel: HETE. Right Panel: BeppoSAX

tion $f\left(L_{\text {iso }}\right) \propto L_{\text {iso }}^{-1}$ [3] (see also [7]). The significance is the fraction of the simulated samples with correlation coefficients that exceed the observed value.

\section{RESULTS}

The results of the analysis of the correlation of $L_{\text {iso }}$ with $1+z$ are reported in table 1 those of the analysis of the correlation of $E_{\text {iso }}$ with $1+z$ are reported in table 2

Threshold effects are clearly playing an important role, and reduce the significance of the evidence for evolution substantially. Nonetheless, after accounting for them, there remains modest ( $5 \%$ significance) evidence for evolution of $E_{\text {iso }}$, and encouraging $\left(9.5 \times 10^{-3}\right.$ significance $)$ evidence for evolution of $L_{\text {iso }}$.

The slopes that we find are typically 3-4. These values are considerably higher than that found by Lloyd-Ronning, Fryer, \& Ramirez-Ruiz [6]. However, our slopes are uncertain, and while our significances are corrected for threshold effects, our slopes are not. The magnitude of this effect remains to be estimated.

\section{DISCUSSION}

If the isotropic-equivalent energies and luminosities of GRBs really do increase with redshift, as the evidence presented here appears to suggest, the consequences for GRB models are profound. Models that rely on variations of the characteristic size of the jet opening angle to produce the observed variations in the isotropic-equivalent energies and luminosities of GRBs (e.g., the uniform jet model [3] ) interpret the correlation as saying that higher- $z$ GRBs have narrower jets. This suggests that the cores of the high- $z$ progenitor stars may rotate more rapidly than those at low $z$. On the other hand, models 
TABLE 1. Correlation Results: $\log \left(L_{\text {iso }}\right)$ vs. $\log (1+z)$

\begin{tabular}{llll}
\hline Quantity & HETE-2 + GRB000131 & BeppoSAX + GRB000131 & Combined \\
\hline Sample Size & 10 & 11 & 20 \\
Correlation Coefficient & 0.87 & 0.83 & 0.76 \\
Formal Significance & $9.0 \times 10^{-4}$ & $1.7 \times 10^{-3}$ & $8.9 \times 10^{-5}$ \\
Threshold-Corrected & & & \\
Significance & $1.2 \times 10^{-2}$ & $4.9 \times 10^{-2}$ & $9.5 \times 10^{-3}$ \\
Slope* $^{*}$ & $3.2 \pm 0.5$ & $4.1 \pm 0.8$ & $3.3 \pm 0.6$ \\
\hline
\end{tabular}

TABLE 2. Correlation Results: $\log \left(E_{\text {iso }}\right)$ vs. $\log (1+z)$

\begin{tabular}{llll}
\hline Quantity & HETE-2 + GRB000131 & BeppoSAX + GRB000131 & Combined \\
\hline Sample Size & 10 & 11 & 20 \\
Correlation Coefficient & 0.74 & 0.75 & 0.66 \\
Formal Significance & $1.3 \times 10^{-2}$ & $8.1 \times 10^{-3}$ & $5.3 \times 10^{-4}$ \\
Threshold-Corrected & & & \\
Significance & $7.8 \times 10^{-2}$ & $1.1 \times 10^{-1}$ & $5.1 \times 10^{-2}$ \\
Slope* & $2.0 \pm 1.0$ & $4.3 \pm 1.1$ & $3.0 \pm 0.8$ \\
\hline
\end{tabular}

* Uncertainties in the slope are $68 \%$ confidence levels.

that rely exclusively on variations of viewing angle to produce the observed variations in the isotropic-equivalent energies and luminosities of GRBs (e.g., the universal jet model) run into difficulty explaining why the viewing angle distribution should change as a function of redshift.

If the isotropic-equivalent energies and luminosities of GRBs really do increase with redshift, GRBs at very high redshifts may be more luminous - and therefore easier to detect - than has been thought, which would make GRBs a more powerful probe of cosmology and the early universe than has been thought.

\section{REFERENCES}

1. Amati, L., et al. 2002, A\&A, 390, 81

2. Band, D. L. 2003, ApJ, 588, 945

3. Donaghy, T., Lamb, D. Q., and Graziani, C. 2003, these proceedings

4. Fenimore, E. E., and Ramirez-Ruiz, E. 2000, ApJ, submitted (astro-ph/0004176)

5. Lamb, D. Q. \& Reichart, D. E. 2000, ApJ, 535, 1

6. Lloyd-Ronning, N. M., Fryer, C. L., and Ramirez-Ruiz, E.2000, ApJ, 574, 554

7. Schmidt, M. 2001, ApJ, 552, 36 